\title{
Quantum phase transitions in the spin-1 Kitaev-Heisenberg chain
}

\author{
Wen-Long You $\odot,{ }^{1,2}$ Gaoyong Sun $\odot,{ }^{1}$ Jie Ren $\odot,{ }^{3}$ Wing Chi Yu $\odot,{ }^{4}$ and Andrzej M. Oleś $\odot^{5,6}$ \\ ${ }^{1}$ College of Science, Nanjing University of Aeronautics and Astronautics, Nanjing 211106, China \\ ${ }^{2}$ School of Physical Science and Technology, Soochow University, Suzhou, Jiangsu 215006, China \\ ${ }^{3}$ Department of Physics, Changshu Institute of Technology, Changshu 215500, China \\ ${ }^{4}$ Department of Physics, City University of Hong Kong, Kowloon, Hong Kong \\ ${ }^{5}$ Institute of Theoretical Physics, Jagiellonian University, Profesora Stanistawa Lojasiewicza 11, PL-30348 Kraków, Poland \\ ${ }^{6}$ Max Planck Institute for Solid State Research, Heisenbergstrasse 1, D-70569 Stuttgart, Germany
}

(Received 20 August 2020; accepted 28 September 2020; published 23 October 2020)

\begin{abstract}
Recently, it has been proposed that higher-spin analogs of the Kitaev interactions $K>0$ may also occur in a number of materials with strong Hund's and spin-orbit coupling. In this work, we use Lanczos diagonalization and density matrix renormalization group methods to investigate numerically the $S=1 \mathrm{Kitaev}-\mathrm{Heisenberg}$ model. The ground-state phase diagram and quantum phase transitions are investigated by employing local and nonlocal spin correlations. We identify two ordered phases at negative Heisenberg coupling $J<0$ : a ferromagnetic phase with $\left\langle S_{i}^{z} S_{i+1}^{z}\right\rangle>0$ and an intermediate left-left-right-right phase with $\left\langle S_{i}^{x} S_{i+1}^{x}\right\rangle \neq 0$. A quantum spin liquid is stable near the Kitaev limit, while a topological Haldane phase is found for $J>0$.
\end{abstract}

DOI: 10.1103/PhysRevB.102.144437

\section{INTRODUCTION}

Kitaev-Heisenberg $(\mathrm{KH})$ models were fostered by an endeavor of achieving the Kitaev physics in transition metal oxides [1]. A continuing interest of bond-directional interactions is motivated by topological quantum computing [2], especially after Kitaev proposed an exactly solvable model of frustrated quantum spins $S=1 / 2$ on a two-dimensional (2D) honeycomb lattice with bond-directional interactions [3]. The Kitaev model was initially treated as a mathematical model describing a topological quantum spin liquid (QSL) ground state (GS) and Majorana excitations, until Jackeli and Khaliullin [4] demonstrated that the bond-directional interactions could be realized in Mott insulators with strong spin-orbit coupling. This innovative concept initiated intense theoretical and experimental search for the $S=1 / 2$ Kitaev QSLs in solid state materials [5]. It has been found that other interactions such as the isotropic Heisenberg and/or off-diagonal exchange terms contribute [6-9], and real systems do not realize the QSL.

The 2D model appears difficult to analyze and its phase diagram has a QSL in the Kitaev limit [10,11], but even the one-dimensional (1D) version of it has several interesting quantum phase transitions (QPTs) [12]. A spin-1/2 1D variant of KH model was defined on a chain, in which two types of nearest-neighbor Kitaev interactions sequentially switch between $S_{i}^{x} S_{i+1}^{x}$ on odd and $S_{i}^{y} S_{i+1}^{y}$ on even bonds next to uniform Heisenberg interactions. The GS phase diagram of spin-1/2

Published by the American Physical Society under the terms of the Creative Commons Attribution 4.0 International license. Further distribution of this work must maintain attribution to the author(s) and the published article's title, journal citation, and DOI. Open access publication funded by the Max Planck Society.
$\mathrm{KH}$ model was depicted using the density matrix renormalization group (DMRG) and exact diagonalization (ED) methods [12]. Much attention has been paid to the Kitaev limit [13-20]. The two-spin correlation functions are found to be extremely short-ranged [21,22], indicating a QSL state.

Recently it was realized that an $S=1 \mathrm{KH}$ model could be designed by considering strong Hund's coupling among two electrons in $e_{g}$ orbitals and strong spin-orbit coupling (SOC) at anion sites [23]. However, relatively little is known about the magnetic properties and particularly the elementary excitation spectrum for higher $S$ on the effect of Heisenberg exchange in the Kitaev chain. It was realized long after Haldane's pioneering work $[24,25]$ that spin models with integer or half-odd integer $S$ are qualitatively different. The Néel state is favored by the Heisenberg antiferromagnetic (AFM) term for half-integer spin $S$, while it cannot play a similar role when $S$ is an integer.

It is recognized that the GS of the $S=1$ Heisenberg antiferromagnet belongs to the Haldane phase, which is separated from all excited states by a finite spin gap [26], and thus two-spin correlation is quenched. The underlying physics of Haldane chains is fairly well understood both in theory and experiments. The Haldane phase of spin-1 XXZ AFM chains was proposed in trapped ions systems [27]. For instance, a hidden $\mathbb{Z}_{2} \times \mathbb{Z}_{2}$ symmetry breaking takes place [28,29] and hence the string order parameters are nonzero in both $x$ and $z$ directions [30,31]. When the Kitaev interaction is taken into account, the spin chains only have a $\mathbb{Z}_{2}$ parity symmetry corresponding to the rotation of $\pi$ around a given axis [32]. If the $\mathbb{Z}_{2}$ symmetry in the GS is broken, whether the string order parameter along the given axis becomes nonzero is unclear [33]. Therefore, it is also an interesting issue to explore the existence of the string correlators in spin-1 chains with lower symmetries than the Heisenberg chain. The phase diagram of the spin-1 generalized Kitaev chain (also dubbed as the 


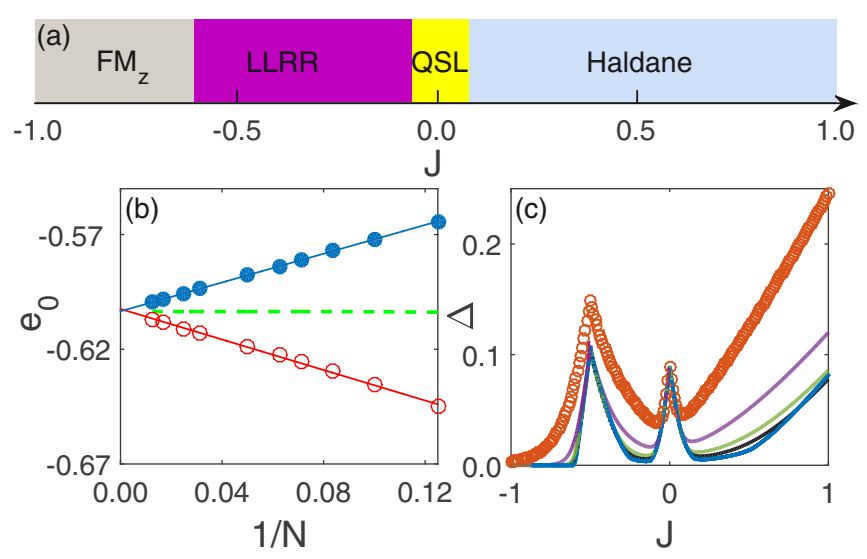

FIG. 1. (a) Schematic phase diagram of the KH model with $K=1$; (b) finite-size scaling of the energy density for $\mathrm{OBC}$ and $J=0$; (c) the energy gap for system sizes ranging from $N=12$ to $N=100$. The blue and red symbols in (b) represent the GS energy of $\hat{H}_{K}$ per site and per bond, respectively. The linear fits correspond to $e_{0}(N)=-0.3326 / N-0.6024$ and $e_{0}(N)=0.3136 / N-0.6034$. The green dashed line is $e_{0}(\infty)$.

compass model in the literature) was also investigated [34]. The GS properties and the low-energy excitations of spin-1 $\mathrm{KH}$ models are elusive and deserve a careful investigation.

The purpose of this paper is twofold. First, we would like to obtain the GS phase diagram and discuss the QPTs in the 1D spin-1 KH model. Second, while some differences in the structure of the invariants between the models with half-odd integer and integer spins have been pointed out [35], the issue of whether there are systematic differences in the nature of the low-energy spectrum is open [36]. The main result of our study is that the GS of the spin-1 KH chain with periodic boundary conditions (PBCs) changes from the QSL to the left-left-right-right (LLRR) (Haldane) phase for $J<0(J>0)$; see Fig. 1(a). Both phases are unique for the $S=11 \mathrm{D} \mathrm{KH}$ model and we employ the ED and the DMRG. In the DMRG simulations, we keep up to $m=500$ eigenstates during the basis truncation and the number of sweeps is $n=30$. These conditions guarantee that the simulation is converged and the truncation error is smaller than $10^{-7}$.

\section{THE MODEL AND ITS PHASE DIAGRAM}

In the present paper we deal with a spin-1 KH chain,

$$
\begin{gathered}
\hat{H}=\hat{H}_{K}+\hat{H}_{J}, \\
\hat{H}_{K}=K \sum_{j=1}^{N / 2}\left(S_{2 j-1}^{x} S_{2 j}^{x}+S_{2 j}^{y} S_{2 j+1}^{y}\right), \\
\hat{H}_{J}=J \sum_{j=1}^{N} \mathbf{S}_{j} \cdot \mathbf{S}_{j+1} .
\end{gathered}
$$

Here $\mathbf{S}_{j}=\left\{S_{j}^{x}, S_{j}^{y}, S_{j}^{z}\right\}$ are the spin-1 operators at site $j$, and $N$ is the total number of sites. The parameters $\{K, J\}$ stand for the Kitaev and Heisenberg exchange coupling. Hereafter, we set $K \equiv 1$. We deal with spin-1 operators in a special representation, $S_{b c}^{\alpha}=i \epsilon_{a b c}$; i.e., $\left\{S^{x}, S^{y}, S^{z}\right\}$ are given by

$$
\left(\begin{array}{ccc}
0 & 0 & 0 \\
0 & 0 & -i \\
0 & i & 0
\end{array}\right), \quad\left(\begin{array}{ccc}
0 & 0 & i \\
0 & 0 & 0 \\
-i & 0 & 0
\end{array}\right), \quad\left(\begin{array}{ccc}
0 & -i & 0 \\
i & 0 & 0 \\
0 & 0 & 0
\end{array}\right) .
$$

Spin operators $\left\{S_{j}^{\alpha}\right\}$ at site $j$ in Eq. (1) obey the SU(2) algebra, $\left[S_{i}^{\alpha}, S_{j}^{\beta}\right]=i \delta_{i j} \epsilon_{\alpha \beta \gamma} S_{j}^{c}$, with the totally antisymmetric tensor $\epsilon$ and $\left(\mathbf{S}_{j}\right)^{2}=S(S+1)=2$.

First we consider the Kitaev limit in Eq. (1), i.e., $J=0$. Then the global spin rotation $\mathrm{SU}(2)$ symmetry is not conserved. We can write the spin operators in $\hat{H}_{K}$ in terms of the ladder operators $S_{j}^{ \pm} \equiv S_{j}^{x} \pm i S_{j}^{y}$, and one finds that $\left[S_{j}^{z}, S_{j}^{ \pm}\right]= \pm S_{j}^{ \pm}$, i.e., the Ising terms in Eq. (2) change the total pseudospin $z$ th component at both odd $x$ link and even $y$ link by either 0 or \pm 2 . A site parity operator is $\Sigma_{j}^{\alpha}=e^{i \pi S_{j}^{\alpha}}$; i.e., $\left\{\Sigma^{x}, \Sigma^{y}, \Sigma^{z}\right\}$ are given by the diagonal matrices that satisfy $\Sigma_{j}^{\alpha}=1-2\left(S_{j}^{\alpha}\right)^{2}$ and $\Sigma_{j}^{x} \Sigma_{j}^{y} \Sigma_{j}^{z}=I$, where $I$ is an identity matrix. The Hamiltonian in Eq. (2) has a global discrete symmetry with respect to rotation by an angle $\pi$ about the $x, y, z$ axes, i.e., $\prod_{j} \Sigma_{j}^{\alpha}$, present in the dihedral group $D_{2}$. The time-reversal symmetry, i.e., $S_{j}^{x, y, z} \rightarrow-S_{j}^{x, y, z}$, and the spatial-inversion symmetry, i.e., $S_{j}^{x, y, z} \rightarrow S_{N+1-j}^{x, y, z}$, are also respected.

Furthermore, one finds that all $\left\{\Sigma_{j}^{\alpha}\right\}$ matrices commute with each other. In addition, $\Sigma_{j}^{\alpha}$ commutes with $S_{j}^{\alpha}$ but anticommutes with $S_{j}^{\beta}(\alpha \neq \beta)$, i.e., $\left\{\Sigma_{j}^{\alpha}, S_{j}^{\beta}\right\}=\left\{\exp \left(i \pi S_{j}^{\alpha}\right), S_{j}^{\beta}\right\}=0$. In this regard, the bond parity operators on odd/even bonds,

$$
W_{2 j-1}=\Sigma_{2 j-1}^{y} \Sigma_{2 j}^{y}, \quad W_{2 j}=\Sigma_{2 j}^{x} \Sigma_{2 j+1}^{x},
$$

define the invariants of the Hamiltonian in Eq. (2) and eigenvalues of $W_{j}$ are \pm 1 . It can be verified that $\left[W_{j}, W_{k}\right]=0$, $\left[W_{j}, \hat{H}_{\mathrm{K}}\right]=0$. The GS of $\hat{H}_{\mathrm{K}}$ lies in the sector with all $W_{j}=1$ which can be proved by applying the reflection positivity technique in the spin-1/2 counterpart [15]. In the GS sector, the system can be mapped to a single qubit-flip model with nearest-neighbor exclusion represented by the effective Hamiltonian [36]:

$$
\tilde{H}_{\mathrm{K}, \mathrm{GS}}=\frac{1}{4} \sum_{j}\left(1-\sigma_{j-1}^{z}\right) \sigma_{j}^{x}\left(1-\sigma_{j+1}^{z}\right) .
$$

At $J=0$ the spectrum is gapped $(\Delta>0)$ and the first excited state is $N$-fold degenerate, corresponding to one $W_{j}=-1$ defect in the $W_{j}=1$ sector. The energy gap is insensitive to the system size, see Fig. 1(c), and remains finite in the thermodynamic limit, $\Delta=0.1764$. Below we show that the Kitaev QSL phase survives in a finite range of coupling $J$.

In the case of open boundary conditions (OBCs) the GS is fourfold degenerate. We consider a finite system with the $\mathrm{OBC}$ in the ED method. It is easy to show that for chains with OBCs the GS energy per site $e_{0}^{l} \equiv E_{0}(N) / N$ [per bond $e_{0}^{u} \equiv E_{0}(N) /(N-1)$ ] gives a lower (upper) bound for the GS energy of an infinite chain [37]. The $N$ dependence of the GS energy $e_{0}(N)=E_{0} / N$ can be fitted to the form $e_{0}(N)=e(\infty)+c / N$, where $c$ is independent of $N$. Indeed, $\log _{10}\left|e_{0}(N)-e(\infty)\right|$ versus $\log _{10} N$ gives $e(\infty) \approx-0.6029$; see Fig. 1(b). 

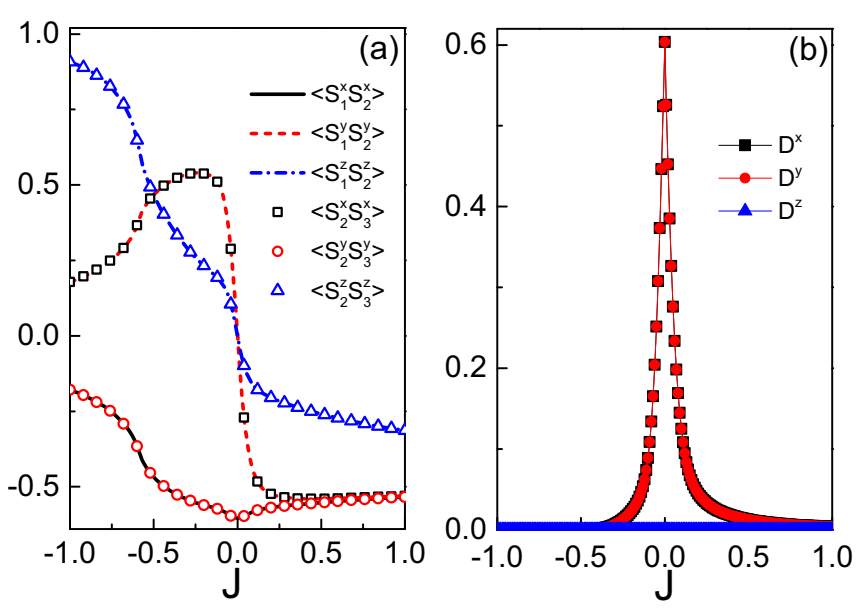

FIG. 2. (a) Two-point spin-spin correlations $C^{\alpha}(i, j)$ (7) and (b) the dimer order parameter $D^{\alpha}(8)$. Parameters: Increasing $J$ and $N=60$.

\section{SPIN-SPIN CORRELATIONS}

According to Ginzburg-Landau theory, a well-defined order parameter is a vital ingredient for characterizing the quantum phase. In order to characterize the Kitaev phase and the QPTs at $J \neq 0$, we calculate the two-point spin correlations,

$$
C^{\alpha}(i, j)=\left\langle S_{i}^{\alpha} S_{j}^{\alpha}\right\rangle, \quad \alpha=x, y, z,
$$

where $i$ and $j$ specify the positions of the initial and final sites, respectively. Accordingly, $r=|j-i|$ measures the separation between the two sites. Similar to its spin- $1 / 2$ counterpart, the two-point correlators precisely vanish beyond nearest neighbors due to the $\mathbb{Z}_{2}$ symmetry, sharing many of the same properties and phenomenology of the spin-1/2 Kitaev honeycomb model [5]. The pure spin-1 Kitaev chain hosts only two nearest-neighbor AFM orders $C^{x}(2 i-1,2 i)$ on $x$ links and $C^{y}(2 i, 2 i+1)$ on $y$ links, as is shown in Fig. 2(a).

Next we turn on a nonzero perturbations $\propto J$, which could undermine the conservation of local quantities characteristic of the Kitaev model. We assume that such interactions are of Heisenberg type, as suggested by possible solid state applications. We then make a comprehensive study on the phase diagram of the $\mathrm{KH}$ Hamiltonian in its full parameter space, using a combination of extensive analytical, DMRG, and ED calculations. It was reported that the spin-1 Kitaev honeycomb model in candidate materials, such as honeycomb $\mathrm{Ni}$ oxides with heavy elements of $\mathrm{Bi}$ and $\mathrm{Sb}$, is accompanied by a finite ferromagnetic (FM) Heisenberg interaction. The Kitaev QSL is stable in the range of $|J| / K>0.08$ [38] and infinitesimal $J$ does not destabilize it.

It is widely recognized that the GS has a qualitative difference between an integer and half-odd integer spin- $S$ models. For $S=1 / 2$, the Kitaev GS is $2^{N / 2-1}$-fold degenerate and such macroscopic degeneracy makes it fragile. Accordingly, an infinitesimal Heisenberg coupling is sufficient to lift the GS degeneracy and to generate magnetic long-range order in the compass-Heisenberg model, either the FM or AFM one [39], when the Heisenberg interactions spoil the $\mathbb{Z}_{2}$ symmetry associated with each bond. It is worth noting that the
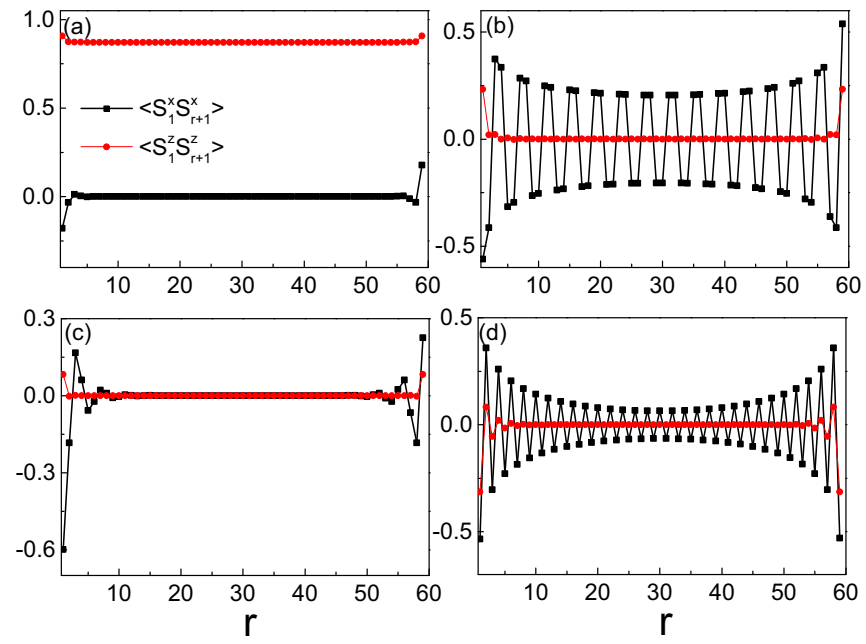

FIG. 3. The correlation between site 1 and site $i$ for increasing distance $r \equiv|i-1|$ for four representative points in different phases (Fig. 1): (a) $J=-1$; (b) $J=-0.2$; (c) $J=-0.03$; and (d) $J=1$. Here we use the PBCs for $N=60$.

low-lying excited-state energy level crossings at $J=0$ take place, which plays an analogous role in the $J_{1}-J_{2}$ Heisenberg chain [40]. Although the second-order derivative of energy density and the normalized fidelity susceptibility exhibit a local peak, the peak declines with increasing system size $N$.

The Hamiltonian is invariant under a rotation around the $z$ axis by an angle $\pi / 2$ (i.e., $S_{j}^{x} \rightarrow S_{j}^{y}, S_{j}^{y} \rightarrow-S_{j}^{x}$ ) and a translation by one lattice site with $i \rightarrow i+1$. The combination of rotation and translation symmetries imply that $C^{x}(1,2)=$ $C^{y}(2,3), C^{y}(1,2)=C^{x}(2,3)$, and $C^{z}(1,2)=C^{z}(2,3)$, which are confirmed in Fig. 2(a). A small Heisenberg coupling can induce other correlations, especially such as $\left\langle S_{2 i-1}^{y} S_{2 i}^{y}\right\rangle$ and $\left\langle S_{2 i}^{x} S_{2 i+1}^{x}\right\rangle$. As shown in Fig. 2(b), the bond-directional order in the Kitaev QSL phase can be captured by the dimer order parameter,

$$
D^{\alpha}=|| C^{\alpha}(2 i-1,2 i)|-| C^{\alpha}(2 i, 2 i+1) \| .
$$

We have verified that the finite-size effects are negligible.

When $J$ varies, the competing correlations will trigger miscellaneous phase transitions. For FM Heisenberg exchange interaction $J<0$, the dominating $x$-component correlations have a negative (positive) sign on odd (even) bonds, evincing that the system develops the LLRR spin order; see Fig. 2(a). This is similar to the GS spin configuration in the ANNNI model, in which the nearest-neighbor interactions favor the FM alignment of neighboring spins while interactions between the next-nearest neighbors foster antiferromagnetism. For $J \simeq-1$, the $C^{z}(i, j)$ correlations dominate and the $\mathrm{FM}_{z}$ GS is found; see Fig. 3(a). When $J$ increases from $J=-1$ to $J=0$, the chain undergoes two successive second-order QPTs at $J_{c 1} \simeq-0.6$ and $J_{c 2} \simeq-0.08$ (Fig. 1). Figure 3 confirms that spin correlations are crucial and identify the QPTs shown in Fig. 1(a). 


\section{TOPOLOGICAL HALDANE PHASE}

On the other hand, the GS of Eq. (3) with AFM couplings $(J>0)$ is a topological phase predicted by Haldane [41], which has a finite excitation gap $\Delta=0.41 \mathrm{~J}$ and exponentially decaying spin correlation functions. More precisely, since the edge states have a finite length for an open chain, the splitting in the lowest energies is exponentially small for longer chains, resulting in fourfold-quasidegenerate GSs below the Haldane gap [42]. It is well known that this phase cannot be characterized by any local symmetry-breaking order parameter. In view of the analogy of GS degeneracy of spin-1 Kitaev and Heisenberg models, a natural question is whether the GS of the Kitaev chain can be adiabatically connected to the Haldane phase without going through a phase transition. The topological nature of the Haldane phase becomes especially clear after Affleck, Kennedy, Lieb, and Tasaki (AKLT) proposed the exactly solvable AKLT model [43], whose GS exhibits intriguing properties, such as a nonlocal string order and $2^{2}$ edge states composed of two free $S=1 / 2$ spinons. Thus, we investigate the string order parameter [30],

$$
O^{\alpha}(l, m)=\left\langle S_{l}^{\alpha} \exp \left(i \pi \sum_{k=l+1}^{m-1} S_{k}^{\alpha}\right) S_{m}^{\alpha}\right\rangle,
$$

whose limiting value $O_{s}^{\alpha}=\lim _{|l-m| \rightarrow \infty}\left\{-O^{\alpha}(l, m)\right\}$ reveals the hidden symmetry breaking, where the $|1\rangle(|-1\rangle)$ states alternate diluted by arbitrary strings of $|0\rangle$. Here $|m\rangle$ is an eigenstate of $S^{\alpha}$ with an eigenvalue $m=-1,0,1$.

Applying the Kennedy-Tasaki (KT) transformation [44], $U_{\mathrm{KT}}=\prod_{j<k} \exp \left(i \pi S_{j}^{z} S_{k}^{x}\right)$, one transforms the diluted AFM phase into the phase containing only $|0\rangle$ and $|1\rangle$ or only $|0\rangle$ and $|-1\rangle$ states, and converts the nonlocal string order into the local FM order. In this regard, Eq. (3) is transformed into a Hamiltonian with short-range interactions, $\tilde{H}_{J}=-J \sum_{i}^{N}\left(S_{i}^{x} S_{i+1}^{x}+S_{i}^{y} S_{i+1}^{y} \Sigma_{i}^{z} \Sigma_{i+1}^{x}+S_{i}^{z} S_{i+1}^{z}\right)$. Note that $\exp \left(i \pi S_{j}^{\alpha}\right) S_{j}^{\alpha}=-S_{j}^{\alpha}$. The KT transformation can transform a Hamiltonian into an equivalent one with a minus sign, which indicates the FM order along either the $x$ or $z$ axis, resulting in $\mathbb{Z}_{2} \times \mathbb{Z}_{2}$ symmetry breaking. In this case, the nonlocal string observable for $\tilde{H}_{J}, O^{\alpha}(l, m)$ in Eq. (9), becomes the two-point correlations $C^{\alpha}(l, m)(\alpha=x, z)$ in Eq. (7) of the transformed Hamiltonian.

Note that in terms of the KT transformation, Eq. (2) is $\tilde{H}_{\mathrm{K}}=-K \sum_{i=1}^{N / 2}\left(S_{2 i-1}^{x} S_{2 i}^{x}+S_{2 i}^{y} S_{2 i+1}^{y} \Sigma_{2 i}^{z} \Sigma_{2 i+1}^{x}\right)$. This suggests that the phase diagram of the KH model with FM Kitaev $K<0$ is similar to the one of the $\mathrm{KH}$ model with AFM Kitaev $K>0$ and transformed spin correlations, i.e., $H(-K,-J) \sim H(K, J)$. Furthermore, the spatial-inversion symmetry, the time-reversal symmetry, and the dihedral $D_{2}$ symmetry are preserved. In this sense, the Haldane phase is still robust as a topological phase and protected by these symmetries $[45,46]$. Finite correlation $C^{z}(1,50)$ or finite string order parameter $O^{x}(1,50)$ between sites 1 and 50 on a $N=100$ lattice with PBC indicate the $\mathrm{FM}_{z}$ (LLRR) phase for $J<J_{c 1}\left(J>J_{c 1}\right)$; see Fig. 4(a). A positive (negative) sign of $O^{x}(1, N / 2)$ for $N=60,100(N=40,80$, not shown $)$ agrees with the periodicity of a multiple of 4 in the LLRR phase. When $J>0$ increases, a QPT occurs from the Kitaev QSL phase to the Haldane phase [47]; see Fig. 4(b). Here finite $x$
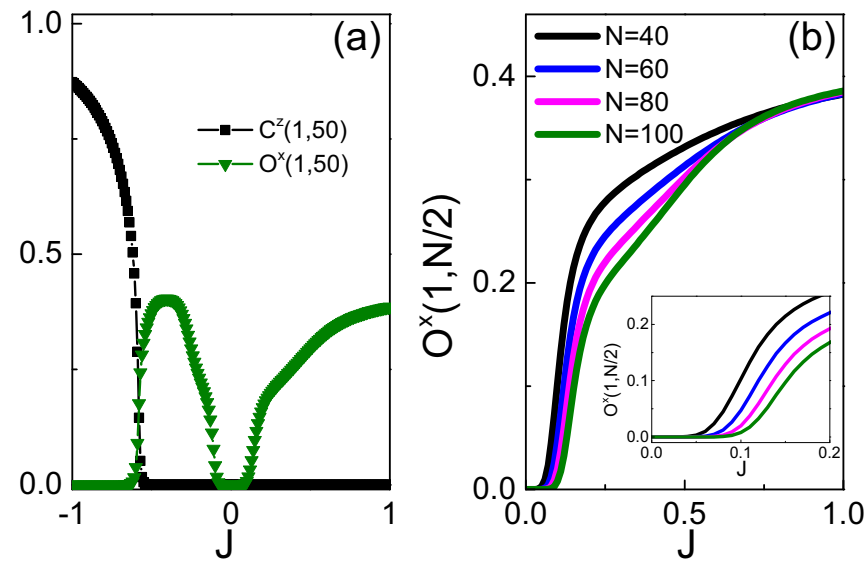

FIG. 4. (a) Two-point correlation $C^{z}$ (7) and the string order parameter $O^{x}$ (9) between sites 1 and 50 for $N=100$ and $J \in(-1,1)$. (b) The string order parameter $O^{x}(9)$ between sites 1 and $N / 2$ in the Haldane phase $(J>0)$. Inset amplifies the region close to $J=0$.

correlations $\left\langle S_{1}^{x} S_{i}^{x}\right\rangle$ occur above $J_{c 3} \approx 0.08$; see Fig. 3(d). The QPT at $J_{c 3}$ is continuous and moves rightward for increasing $N$; see the inset of Fig. 4(b). A more precise determination of the stability range of the QSL and of the critical point $J_{c 3}$ requires calculations on larger systems.

\section{SUMMARY AND CONCLUSIONS}

In summary, we characterize the ground-state properties of the Kitaev-Heisenberg $S=1$ chain by the local and nonlocal correlations and identify four distinct phases for $J \in(-1,1)$. For large negative $J$, the $\mathrm{FM}_{z}$ order is favored; increasing value of $J$ gives a transition to an intermediate LLRR phase. These spin correlations vanish beyond the second phase transition when $J$ approaches the Kitaev limit. In stark contrast to the gapless QSL of the $S=1 / 2 \mathrm{Kitaev}$ chain, the $S=1$ chain supports a gapped QSL near $J=0$. It is characterized by the short-range correlations and the dimer order parameter (8). Further increase of $J$ suppresses the dimer order and gives a valence bond solid with the singlets oriented along the $x$ direction as inferred from finite string order parameter $O^{x}$, the Haldane phase. It is robust against the Kitaev interactions since it is protected by the combination of spatial-inversion symmetry, time-reversal symmetry, and dihedral $D_{2}$ symmetry. It maintains its topological character in a range of $J>0$, and cannot evolve adiabatically to other phases.

The ground-state properties in the presence of anisotropy in the Kitaev interactions and Heisenberg exchange interactions deserve further studies. It is also an interesting future issue to investigate possible phase transitions caused by the effect of non-Kitaev interactions, such as off-diagonal exchange interactions, which have been extensively investigated in a few candidate materials of the Kitaev magnets: $\mathrm{A}_{2} \mathrm{IrO}_{3}$ [6], $\alpha-\mathrm{RuCl}_{3}$ [48-54], $\beta$ - $\mathrm{LiIrO}_{3}$ [55-58], and $\mathrm{K}_{2} \mathrm{IrO}_{3}$ [59].

Note added in proof: A recent study of the 2D spin-1 Kitaev-Heisenberg model on the honeycomb lattice [60] shows that, similar to the $1 \mathrm{D}$ model reported here, a competition occurs between quantum spin liquid and symmetry broken phases. 


\section{ACKNOWLEDGMENTS}

This work is supported by the National Natural Science Foundation of China (NSFC) under Grants No. 11704186 and No. 11474211 and by the National Science Centre (NCN, Poland) under Project No. 2016/23/B/ST3/00839.
W.-L.Y. acknowledges support by the startup fund (Grant No. 1008-YAH20006) of Nanjing University of Aeronautics and Astronautics. W.C.Y. acknowledges financial support by City University of Hong Kong through Grant No. 9610438. A.M.O. is grateful for support from an Alexander von Humboldt Foundation (Humboldt-Forschungspreis).
[1] Z. Nussinov and J. van den Brink, Compass models: Theory and physical motivations, Rev. Mod. Phys. 87, 1 (2015).

[2] D. Aasen, R. S. K. Mong, B. M. Hunt, D. Mandrus, and J. Alicea, Electrical Probes of the Non-Abelian Spin Liquid in Kitaev Materials, Phys. Rev. X 10, 031014 (2020).

[3] A. Y. Kitaev, Fault-tolerant quantum computation by anyons, Ann. Phys. (Amsterdam) 303, 2 (2003); A. Kitaev, Anyons in an exactly solved model and beyond, ibid. 321, 2 (2006).

[4] G. Jackeli and G. Khaliullin, Mott Insulators in the Strong SpinOrbit Coupling Limit: From Heisenberg to a Quantum Compass and Kitaev Models, Phys. Rev. Lett. 102, 017205 (2009).

[5] S. M. Winter, A. A. Tsirlin, M. Daghofer, J. van den Brink, Y. Singh, P. Gegenwart, and R. Valenti, Models and materials for generalized Kitaev magnetism, J. Phys.: Condens. Matter 29, 493002 (2017).

[6] J. G. Rau, E. Kin-Ho Lee, and H.-Y. Kee, Generic Spin Model for the Honeycomb Iridates beyond the Kitaev Limit, Phys. Rev. Lett. 112, 077204 (2014).

[7] J. Rusnačko, D. Gotfryd, and J. Chaloupka, Kitaev-like honeycomb magnets: Global phase behavior and emergent effective models, Phys. Rev. B 99, 064425 (2019).

[8] W. Yang, A. Nocera, T. Tummuru, H.-Y. Kee, and I. Affleck, Phase Diagram of the Spin-1/2 Kitaev-Gamma Chain and Emergent SU(2) Symmetry, Phys. Rev. Lett. 124, 147205 (2020).

[9] W. Yang, A. Nocera, and Ian Affleck, Comprehensive study of the phase diagram of the spin- $\frac{1}{2}$ Kitaev-Heisenberg-Gamma chain, Phys. Rev. Research 2, 033268 (2020); Spin wave theory of one-dimensional generalized Kitaev model, Phys. Rev. B 102, 134419 (2020).

[10] J. Chaloupka, G. Jackeli, and G. Khaliullin, Zigzag Magnetic Order in the Iridium Oxide $\mathrm{Na}_{2} \mathrm{IrO}_{3}$, Phys. Rev. Lett. 110, 097204 (2013).

[11] D. Gotfryd, J. Rusnačko, K. Wohlfeld, G. Jackeli, J. Chaloupka, and A. M. Oleś, Phase diagram and spin correlations of the Kitaev-Heisenberg model: Importance of quantum effects, Phys. Rev. B 95, 024426 (2017).

[12] C. E. Agrapidis, J. van den Brink, and S. Nishimoto, Ordered states in the Kitaev-Heisenberg model: From 1D chains to 2D honeycomb, Sci. Rep. 8, 1815 (2018).

[13] W. Brzezicki, J. Dziarmaga, and A. M. Oleś, Quantum phase transition in the one-dimensional compass model, Phys. Rev. B 75, 134415 (2007).

[14] W. Brzezicki and A. M. Oleś, Quantum Phase Transition in the One-Dimensional XZ Model, Acta Phys. Pol. A 115, 162 (2009).

[15] W.-L. You and G.-S. Tian, Quantum phase transition in the onedimensional compass model using the pseudospin approach, Phys. Rev. B 78, 184406 (2008).

[16] W.-L. You, G.-S. Tian, and H.-Q. Lin, The low-energy states and directional long-range order in the two-dimensional quan- tum compass model, J. Phys. A: Math. Theor. 43, 275001 (2010).

[17] P.-S. He, W.-L. You, and G.-S. Tian, Two-dimensional quantum compass model in a staggered field: Some rigorous results, Chin. Phys. B 20, 017503 (2011).

[18] E. Eriksson and H. Johannesson, Multicriticality and entanglement in the one-dimensional quantum compass model, Phys. Rev. B 79, 224424 (2009).

[19] V. Subrahmanyam, Block entropy for Kitaev-type spin chains in a transverse field, Phys. Rev. A 88, 032315 (2013).

[20] G.-H. Liu, W. Li, W.-L. You, G.-S. Tian, and G. Su, Matrix product state and quantum phase transitions in the onedimensional extended quantum compass model, Phys. Rev. B 85, 184422 (2012).

[21] G. Baskaran, S. Mandal, and R. Shankar, Exact Results for Spin Dynamics and Fractionalization in the Kitaev Model, Phys. Rev. Lett. 98, 247201 (2007).

[22] W.-L. You, Quantum correlation in one-dimensional extended quantum compass model, Eur. Phys. J. B 85, 83 (2012).

[23] P. P. Stavropoulos, D. Pereira, and H.-Y. Kee, Microscopic Mechanism for a Higher-Spin Kitaev Model, Phys. Rev. Lett. 123, 037203 (2019).

[24] F. D. M. Haldane, Continuum dynamics of the 1-D Heisenberg antiferromagnet: Identification with the $\mathrm{O}(3)$ nonlinear sigma model, Phys. Lett. A 93, 464 (1983).

[25] F. D. M. Haldane, Nonlinear Field Theory of Large-Spin Heisenberg Antiferromagnets: Semiclassically Quantized Solitons of the One-Dimensional Easy-Axis Néel State, Phys. Rev. Lett. 50, 1153 (1983).

[26] S. R. White and D. A. Huse, Numerical renormalization-group study of low-lying eigenstates of the antiferromagnetic $S=1$ Heisenberg chain, Phys. Rev. B 48, 3844 (1993).

[27] I. Cohen and A. Retzker, Proposal for Verification of the Haldane Phase Using Trapped Ions, Phys. Rev. Lett. 112, 040503 (2014).

[28] T. Kennedy and H. Tasaki, Hidden $\mathbb{Z}_{2} \times \mathbb{Z}_{2}$ symmetry breaking in Haldane-gap antiferromagnets, Phys. Rev. B 45, 304 (1992).

[29] S. Takada and K. Kubo, Nonlocal unitary transformations on $S=1$ antiferromagnetic spin chains, J. Phys. Soc. Jpn. 60, 4026 (1991).

[30] M. den Nijs and K. Rommelse, Preroughening transitions in crystal surfaces and valence-bond phases in quantum spin chains, Phys. Rev. B 40, 4709 (1989).

[31] H. Tasaki, Quantum Liquid in Antiferromagnetic Chains: A Stochastic Geometric Approach to the Haldane Gap, Phys. Rev. Lett. 66, 798 (1991).

[32] E. Berg, E. G. Dalla Torre, T. Giamarchi, and E. Altman, Rise and fall of hidden string order of lattice bosons, Phys. Rev. B 77, 245119 (2008). 
[33] Y. Hatsugai and M. Kohmoto, Numerical study of the hidden antiferromagnetic order in the Haldane phase, Phys. Rev. B 44, 11789 (1991).

[34] G.-H. Liu, L.-J. Kong, and W.-L. You, Quantum phase transitions in spin-1 compass chains, Eur. Phys. J. B 88, 284 (2015)

[35] G. Baskaran, D. Sen, and R. Shankar, Spin-S Kitaev model: Classical ground states, order from disorder, and exact correlation functions, Phys. Rev. B 78, 115116 (2008).

[36] D. Sen, R. Shankar, D. Dhar, and K. Ramola, Spin-1 Kitaev model in one dimension, Phys. Rev. B 82, 195435 (2010).

[37] W.-L. You, G.-S. Tian, and H.-Q. Lin, Existence of long-range orbital order in a two-dimensional orbital-only model, Phys. Rev. B 75, 195118 (2007).

[38] C. Hickey, C. Berke, P. P. Stavropoulos, H.-Y. Kee, and $\mathrm{S}$. Trebst, Field-driven gapless spin liquid in the spin-1 Kitaev honeycomb model, Phys. Rev. Research 2, 023361 (2020).

[39] F. Trousselet, A. M. Oleś, and P. Horsch, Compass-Heisenberg model on the square lattice-Spin order and elementary excitations, Europhys. Lett. 91, 40005 (2010); Magnetic properties of nanoscale compass-Heisenberg planar clusters, Phys. Rev. B 86, 134412 (2012).

[40] S. Chen, Li Wang, S.-J. Gu, and Y. Wang, Fidelity and quantum phase transition for the Heisenberg chain with next-nearestneighbor interaction, Phys. Rev. E 76, 061108 (2007).

[41] Y. Yang, S.-J. Ran, Xi Chen, Z.-Z. Sun, S.-S. Gong, Z. Wang, and G. Su, Reentrance of the topological phase in a spin1 frustrated Heisenberg chain, Phys. Rev. B 101, 045133 (2020).

[42] T. Kennedy, Exact diagonalisations of open spin-1 chains, J. Phys.: Condens. Matter 2, 5737 (1990).

[43] I. Affleck, T. Kennedy, E. H. Lieb, and H. Tasaki, Rigorous Results on Valence-Bond Ground States in Antiferromagnets, Phys. Rev. Lett. 59, 799 (1987).

[44] M. Oshikawa, Hidden $\mathbb{Z}_{2} \times \mathbb{Z}_{2}$ symmetry in quantum spin chains with arbitrary integer spin, J. Phys.: Condens. Matter 4, 7469 (1992).

[45] F. Pollmann, A. M. Turner, E. Berg, and M. Oshikawa, Entanglement spectrum of a topological phase in one dimension, Phys. Rev. B 81, 064439 (2010).

[46] F. Pollmann, E. Berg, A. M. Turner, and M. Oshikawa, Symmetry protection of topological phases in one-dimensional quantum spin systems, Phys. Rev. B 85, 075125 (2012).

[47] J. Ren, W.-L. You, and A. M. Oleś, Quantum phase transitions in a spin-1 antiferromagnetic chain with long-range interactions and modulated single-ion anisotropy, Phys. Rev. B 102, 024425 (2020).
[48] D. Takikawa and S. Fujimoto, Impact of off-diagonal exchange interactions on the Kitaev spin-liquid state of $\alpha-\mathrm{RuCl}_{3}$, Phys. Rev. B 99, 224409 (2019).

[49] L. Janssen, E. C. Andrade, and M. Vojta, Magnetization processes of zigzag states on the honeycomb lattice: Identifying spin models for $\alpha-\mathrm{RuCl}_{3}$ and $\mathrm{Na}_{2} \mathrm{IrO}_{3}$, Phys. Rev. B 96, 064430 (2017).

[50] W. Wang, Z.-Y. Dong, S.-Li Yu, and J.-X. Li, Theoretical investigation of magnetic dynamics in $\alpha-\mathrm{RuCl}_{3}$, Phys. Rev. B 96 , 115103 (2017).

[51] P. Lampen-Kelley, S. Rachel, J. Reuther, J.-Q. Yan, A. Banerjee, C. A. Bridges, H. B. Cao, S. E. Nagler, and D. Mandrus, Anisotropic susceptibilities in the honeycomb Kitaev system $\alpha-\mathrm{RuCl}_{3}$, Phys. Rev. B 98, 100403(R) (2018).

[52] I. O. Ozel, C. A. Belvin, E. Baldini, I. Kimchi, S. Do, K.-Y. Choi, and N. Gedik, Magnetic field-dependent lowenergy magnon dynamics in $\alpha-\mathrm{RuCl}_{3}$, Phys. Rev. B 100, 085108 (2019).

[53] R. L. Smit, S. Keupert, O. Tsyplyatyev, P. A. Maksimov, A. L. Chernyshev, and P. Kopietz, Magnon damping in the zigzag phase of the Kitaev-Heisenberg- $\Gamma$ model on a honeycomb lattice, Phys. Rev. B 101, 054424 (2020).

[54] K. Ran, J. Wang, W. Wang, Z.-Y. Dong, X. Ren, S. Bao, S. Li, Z. Ma, Y. Gan, Y. Zhang et al., Spin-Wave Excitations Evidencing the Kitaev Interaction in Single Crystalline $\alpha-\mathrm{RuCl}_{3}$, Phys. Rev. Lett. 118, 107203 (2017).

[55] S. Ducatman, I. Rousochatzakis, and N. B. Perkins, Magnetic structure and excitation spectrum of the hyperhoneycomb Kitaev magnet $\beta$ - $\mathrm{Li}_{2} \mathrm{IrO}_{3}$, Phys. Rev. B 97, 125125 (2018).

[56] I. Rousochatzakis and N. B. Perkins, Classical Spin Liquid Instability Driven by Off-Diagonal Exchange in Strong SpinOrbit Magnets, Phys. Rev. Lett. 118, 147204 (2017); Magnetic field induced evolution of intertwined orders in the Kitaev magnet $\beta$ - $\mathrm{Li}_{2} \mathrm{IrO}_{3}$, Phys. Rev. B 97, 174423 (2018).

[57] M. Majumder, R.S. Manna, G. Simutis, J.C. Orain, T. Dey, F. Freund, A. Jesche, R. Khasanov, P.K. Biswas, E. Bykova et al., Breakdown of Magnetic Order in the Pressurized Kitaev Iridate $\beta$ - $\mathrm{Li}_{2} \mathrm{IrO}_{3}$, Phys. Rev. Lett. 120, 237202 (2018).

[58] H.-S. Kim, Y. B. Kim, and H.-Y. Kee, Revealing frustrated local moment model for pressurized hyperhoneycomb iridate: Paving the way toward a quantum spin liquid, Phys. Rev. B 94, 245127 (2016).

[59] R. Yadav, S. Nishimoto, M. Richter, J. van den Brink, and R. Ray, Large off-diagonal exchange couplings and spin liquid states in $C_{3}$-symmetric iridates, Phys. Rev. B 100, 144422 (2019).

[60] X.-Y. Dong, and D. N. Sheng, Spin-1 Kitaev-Heisenberg model on a honeycomb lattice, Phys. Rev. B 102, 121102(R) (2020). 